Network Working Group

Request for Comments: 3081

Category: Standards Track

M. Rose

Invisible Worlds, Inc.

March 2001

Mapping the BEEP Core onto TCP

Status of this Memo

This document specifies an Internet standards track protocol for the Internet community, and requests discussion and suggestions for improvements. Please refer to the current edition of the "Internet Official Protocol Standards" (STD 1) for the standardization state and status of this protocol. Distribution of this memo is unlimited.

Copyright Notice

Copyright (C) The Internet Society (2001). All Rights Reserved.

Abstract

This memo describes how a BEEP (Blocks Extensible Exchange Protocol) session is mapped onto a single TCP (Transmission Control Protocol) connection.

Table of Contents

1. Introduction . . . . . . . . . . . . . . . . . . . . . . . . 1

2. Session Management . . . . . . . . . . . . . . . . . 2

3. Message Exchange . . . . . . . . . . . . . . . . . . 2

3.1 Flow Control. . . . . . . . . . . . . . . . . . . 3

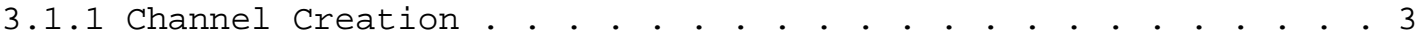

3.1.2 Sending Messages . . . . . . . . . . . . . . . . . . 3

3.1.3 Processing SEQ Frames . . . . . . . . . . . . . . . . . . 4

3.1.4 Use of Flow Control . . . . . . . . . . . . . . . . . 4

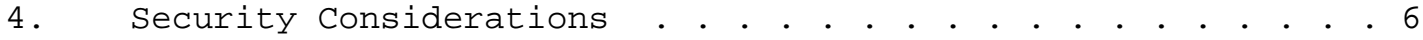

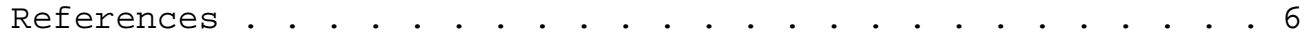
Author's Address . . . . . . . . . . . . . . . . 6

A. Acknowledgements . . . . . . . . . . . . . . . . . . . . 7

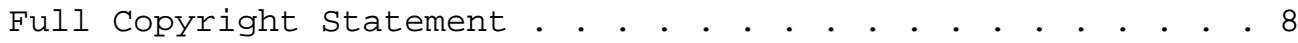

1. Introduction

This memo describes how a BEEP [1] session is mapped onto a single TCP [2] connection. Refer to section 2.5 of [1] for an explanation of the mapping requirements. 


\section{Session Management}

The mapping of BEEP session management onto the TCP service is straight-forward.

A BEEP session is established when a TCP connection is established between two BEEP peers:

- the BEEP peer that issues a passive TCP OPEN call is termed the listener; and,

- the BEEP peer that issues an active TCP OPEN call is termed the initiator.

A simultaneous TCP OPEN would result in both BEEP peers believing they are the initiator and neither peer will be able to start any channels. Because of this, services based on BEEP must be designed so that simultaneous TCP OPENs cannot occur.

If both peers agree to release a BEEP session (c.f., [1]'s Section 2.4), the peer sending the "ok" reply, immediately issues the TCP CLOSE call. Upon receiving the reply, the other peer immediately issues the TCP CLOSE call.

A BEEP session is terminated when either peer issues the TCP ABORT call, and the TCP connection is subsequently aborted.

3. Message Exchange

The mapping of BEEP exchanges onto the TCP service is less straightforward.

Messages are reliably sent and received using TCP's SEND and RECEIVE calls. (This also provides ordered delivery of messages on the same channel.)

Although TCP imposes flow control on a per-connection basis, if multiple channels are simultaneously in use on a BEEP session, BEEP must provide a mechanism to avoid starvation and deadlock. To achieve this, BEEP re-introduces a mechanism used by the TCP: window-based flow control -- each channel has a sliding window that indicates the number of payload octets that a peer may transmit before receiving further permission. 


\subsection{Flow Control}

Recall from Section 2.2.1.2 of [1] that every payload octet sent in each direction on a channel has an associated sequence number. Numbering of payload octets within a data frame is such that the first payload octet is the lowest numbered, and the following payload octets are numbered consecutively.

The actual sequence number space is finite, though very large, ranging from $0 . .4294967295(2 * * 32-1)$. Since the space is finite, all arithmetic dealing with sequence numbers is performed modulo $2 \star \star 32$. This unsigned arithmetic preserves the relationship of sequence numbers as they cycle from $2 * * 32-1$ to 0 again. Consult Sections 2 through 5 of [3] for a discussion of the arithmetic properties of sequence numbers.

\subsubsection{Channel Creation}

When a channel is created, the sequence number associated with the first payload octet of the first data frame is 0 , and the initial window size for that channel is 4096 octets. After channel creation, a BEEP peer may update the window size by sending a SEQ frame (Section 3.1.3).

If a BEEP peer is asked to create a channel and it is unable to allocate at least 4096 octets for that channel, it must decline creation of the channel, as specified in section 2.3.1.2 of [1]. Similarly, during establishment of the BEEP session, if the BEEP peer acting in the listening role is unable to allocate at least 4096 octets for channel 0 , then it must return a negative reply, as specified in Section 2.4 of [1], instead of a greeting.

\subsubsection{Sending Messages}

Before a message is sent, the sending BEEP peer must ensure that the size of the payload is within the window advertised by the receiving BEEP peer. If not, it has three choices:

o if the window would allow for at least one payload octet to be sent, the BEEP peer may segment the message and start by sending a smaller data frame (up to the size of the remaining window);

- the BEEP peer may delay sending the message until the window becomes larger; or, 
- the BEEP peer may signal to its application that it is unable to send the message, allowing the application to try again at a later time (or perhaps signaling its application when a larger window is available).

The choice is implementation-dependent, although it is recommended that the application using BEEP be given a mechanism for influencing the decision.

\subsubsection{Processing SEQ Frames}

As an application accepts responsibility for incoming data frames, its BEEP peer should send SEQ frames to advertise a new window.

The ABNF [4] for a SEQ frame is:

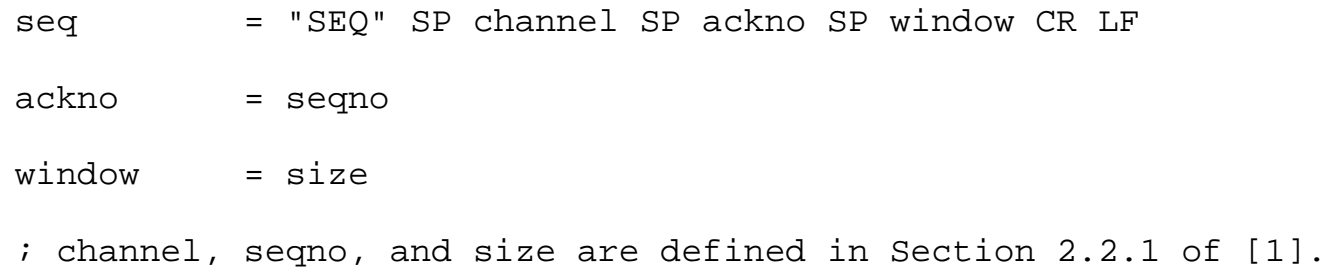

o a channel number;

- an acknowledgement number, that indicates the value of the next sequence number that the sender is expecting to receive on this channel; and,

- a window size, that indicates the number of payload octets beginning with the one indicated by the acknowledgement number that the sender is expecting to receive on this channel.

A single space character (decimal code 32," ") separates each component. The SEQ frame is terminated with a CRLF pair.

When a SEQ frame is received, if any of the channel number, acknowledgement number, or window size cannot be determined or is invalid, then the BEEP session is terminated without generating a response, and it is recommended that a diagnostic entry be logged.

\subsubsection{Use of Flow Control}

The key to successful use of flow control within BEEP is to balance performance and fairness: 
- large messages should be segmented into frames no larger than two-thirds of $\mathrm{TCP}^{\prime} \mathrm{s}$ negotiated maximum segment size;

- frames for different channels with traffic ready to send should be sent in a round-robin fashion;

- each time a frame is received, a SEQ frame should be sent whenever the window size that will be sent is at least one half of the buffer space available to this channel; and,

- if the transport service presents multiple frames to a BEEP peer simultaneously, then a single consolidating SEQ frame may be sent.

In order to avoid pathological interactions with the transport service, it is important that a BEEP peer advertise windows based on available buffer space, to allow data to be read from the transport service as soon as available. Further, SEQ frames for a channel must have higher priority than messages for that channel.

Implementations may wish to provide queue management facilities to the application using BEEP, e.g., channel priorities, (relative) buffer allocations, and so on. In particular, implementations should not allow a given channel to monopolize the underlying transport window (e.g., slow readers should get small windows).

In addition, where possible, implementations should support transport layer APIs that convey congestion information. These APIs allow an implementation to determine its share of the available bandwidth, and also be notified of changes in the estimated path bandwidth. Note that when a BEEP session has multiple channels that are simultaneously exchanging large messages, implementations without access to this information may have uncertain fairness and progress properties during times of network congestion.

Finally, implementors should follow the guidelines given in the relevant portions of RFC1122 [5] that deal with flow control (and bear in mind that issues such as retransmission, while they interact with flow control in TCP, are not applicable to this memo). For example, Section 4.2.2.16 of RFC1122 [5] indicates that a "receiver SHOULD NOT shrink the window, i.e., move the right window edge to the left" and then discusses the impact of this rule on unacknowledged data. In the context of mapping BEEP onto a single TCP connection, only the portions concerning flow control should be implemented. 
4. Security Considerations

Consult Section [1]'s Section 9 for a discussion of security issues. References

[1] Rose, M., "The Blocks Extensible Exchange Protocol Core", RFC 3080 , March 2001 .

[2] Postel, J., "Transmission Control Protocol", STD 7, RFC 793, September 1981 .

[3] Elz, R. and R. Bush, "Serial Number Arithmetic", RFC 1982, August 1996 .

[4] Crocker, D. and P. Overell, "Augmented BNF for Syntax Specifications: ABNF", RFC 2234, November 1997.

[5] Braden, R., "Requirements for Internet Hosts -- Communication Layers", STD 3, RFC 1122, October 1989.

Author's Address

Marshall T. Rose

Invisible Worlds, Inc.

1179 North McDowell Boulevard

Petaluma, CA 94954-6559

US

Phone: +1707 7893700

EMail: mrose@invisible.net

URI: http://invisible.net/ 
Appendix A. Acknowledgements

The author gratefully acknowledges the contributions of: Dave Crocker, Steve Harris, Eliot Lear, Keith McCloghrie, Craig Partridge, Vernon Schryver, and, Joe Touch. In particular, Dave Crocker provided helpful suggestions on the nature of flow control in the mapping. 
Full Copyright statement

Copyright (C) The Internet Society (2001). All Rights Reserved.

This document and translations of it may be copied and furnished to others, and derivative works that comment on or otherwise explain it or assist in its implementation may be prepared, copied, published and distributed, in whole or in part, without restriction of any kind, provided that the above copyright notice and this paragraph are included on all such copies and derivative works. However, this document itself may not be modified in any way, such as by removing the copyright notice or references to the Internet society or other Internet organizations, except as needed for the purpose of developing Internet standards in which case the procedures for copyrights defined in the Internet Standards process must be followed, or as required to translate it into languages other than English.

The limited permissions granted above are perpetual and will not be revoked by the Internet society or its successors or assigns.

This document and the information contained herein is provided on an "AS IS" basis and THE INTERNET SOCIETY AND THE INTERNET ENGINEERING TASK FORCE DISCLAIMS ALL WARRANTIES, EXPRESS OR IMPLIED, INCLUDING BUT NOT LIMITED TO ANY WARRANTY THAT THE USE OF THE INFORMATION HEREIN WILL NOT INFRINGE ANY RIGHTS OR ANY IMPLIED WARRANTIES OF MERCHANTABILITY OR FITNESS FOR A PARTICULAR PURPOSE.

Acknowledgement

Funding for the RFC Editor function is currently provided by the Internet society. 